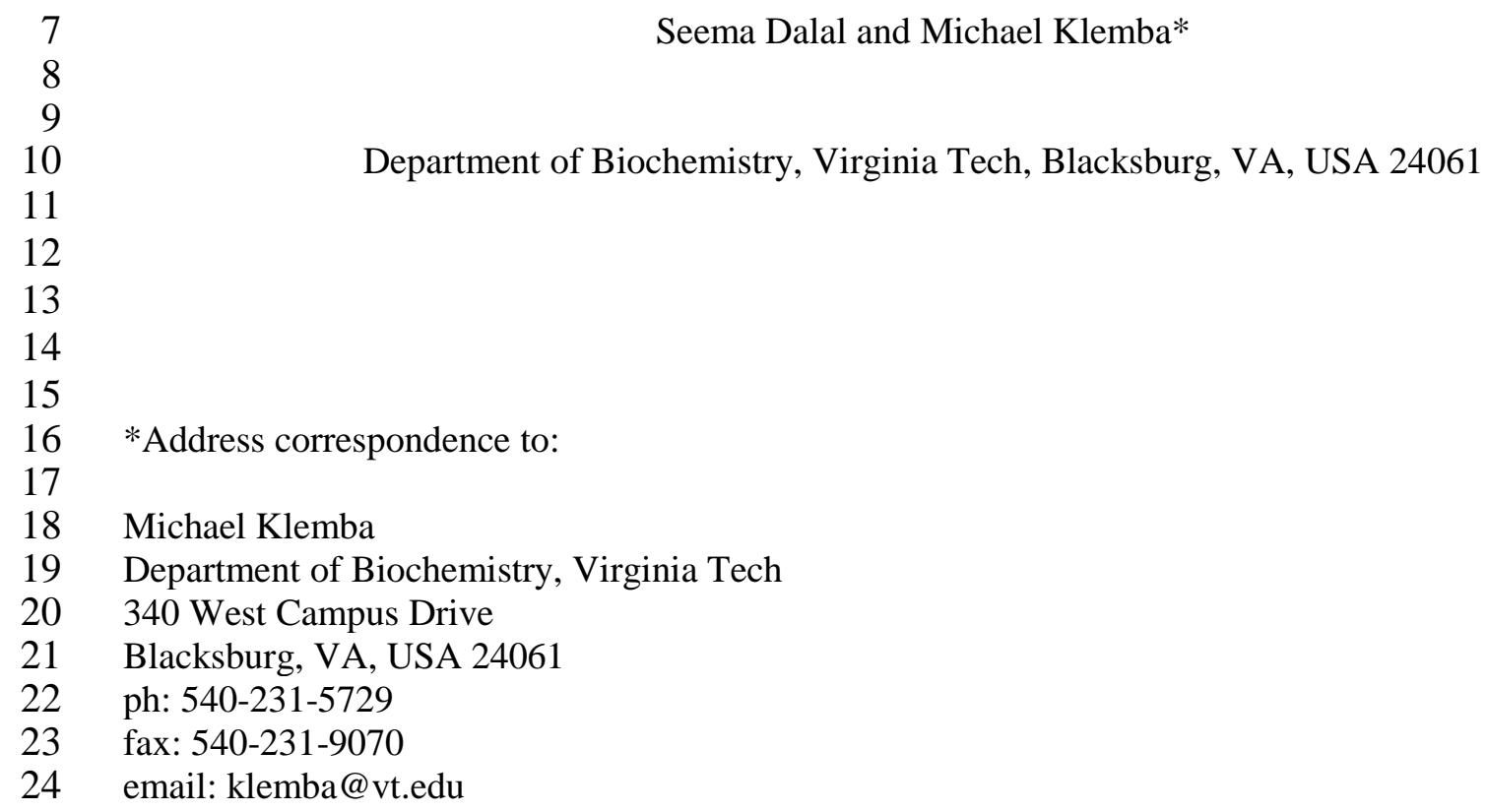

*Address correspondence to:

Department of Biochemistry, Virginia Tech, Blacksburg, VA, USA 24061

Seema Dalal and Michael Klemba*

Michael Klemba

Department of Biochemistry, Virginia Tech

340 West Campus Drive

21 Blacksburg, VA, USA 24061

22 ph: 540-231-5729

23 fax: 540-231-9070

24 email: klemba@vt.edu 
ABSTRACT

The endocytosis and catabolism of large quantities of host cell hemoglobin is a hallmark of the

33 intraerythrocytic asexual stage of the human malaria parasite Plasmodium falciparum. It is known that the

34 parasite's production of amino acids from hemoglobin far exceeds its metabolic needs. Here, we show

35 that $P$. falciparum effluxes large quantities of certain non-polar (Ala, Leu, Val, Pro, Phe, Gly) and polar

36 (Ser, Thr, His) amino acids to the external medium. That these amino acids originate from hemoglobin

37 catabolism is indicated by the strong correlation between individual amino acid efflux rates and their

38 abundances in hemoglobin, and the ability of the food vacuole falcipain inhibitor E-64d to greatly

39 suppress efflux rates. We then developed a rapid, sensitive and precise method for quantifying flux

40 through the hemoglobin endocytic-catabolic pathway that is based on leucine efflux. Optimization of the

41 method involved the generation of a novel amino acid-restricted RPMI formulation as well as the

42 validation of $D$-norvaline as an internal standard. The utility of this method was demonstrated by

43 characterizing the effects of the phosphatidylinositol-3-kinase inhibitors wortmannin and

44 dihydroartemisinin on the kinetics of Leu efflux. Both compounds rapidly inhibited Leu efflux, which is

45 consistent with a role for phosphtidylinositol-3-phosphate production in the delivery of hemoglobin to the

46 food vacuole; however, wortmannin inhibition was transient, which was likely due to the instability of

47 this compound in culture medium. The simplicity, convenience and non-invasive nature of the Leu efflux

48 assay described here makes it ideal for characterizing the in vivo kinetics of hemoglobin endocytosis and

49 catabolism, for inhibitor target validation studies, and for medium-throughput screens to identify novel

50 inhibitors of cytostomal endocytosis.

Keywords: malaria, hemoglobin, amino acid, phosphatidylinositol-3-kinase, wortmannin, dihydroartemisinin

\section{Abbreviations:}

57 DHA, dihydroartemisinin; iRBC, infected red blood cell; Nva, $D$-norvaline; PI3P, phosphatidylinositol-3phosphate; PI3K, phosphatidylinositol-3-kinase; UPLC, ultra-high pressure liquid chromatography;

60 uRBC, uninfected red blood cell. 


\section{INTRODUCTION}

Asexual replication of Plasmodium spp. within human erythrocytes gives rise to the clinical

63 symptoms of malaria. During its intraerythrocytic residence, $P$. falciparum, the most virulent human

64 malaria species, degrades up to three-quarters of the host erythrocyte's hemoglobin [1, 2]. Hemoglobin

65 endocytosis takes place at the cytostome, a specialized pore-like structure of unknown composition that is

66 associated with invaginating parasitophorous vacuole and parasite plasma membranes [3-6]. Catabolism

67 of hemoglobin occurs in the food vacuole, an acidic organelle that is formed through the fusion of

68 endocytic structures beginning at about 14 hours post-invasion [3]. The mechanistic details of

69 hemoglobin transport from the cytostome to the food vacuole remain obscure. There is some experimental

70 support for a model for hemoglobin transport in which an unconventional double-membrane transport

71 vesicle pinches off from the cytostome and fuses with the food vacuole membrane [3, 5-8]. Potential non-

72 cytostomal phagotropic structures have also been observed [5, 8]; however, the extent of their

73 contribution to hemoglobin endocytosis remains to be established.

The food vacuole contains endo- and exo-peptidases that catalyze the hydrolysis of $\alpha$ - and $\beta$ globin to amino acids [9-11]. The quantities of amino acids generated through hemoglobin catabolism are

76 sufficient to satisfy the parasite's needs for protein synthesis and other metabolic activities requiring

77 amino acids; only isoleucine, which is absent from human hemoglobin, needs to be obtained from an

78 exogenous source [12]. Intriguingly, only about one-fifth of globin-derived amino acids are used by $P$.

79 falciparum [2]. Studies with intraerythrocytic malaria parasites have revealed that substantial quantities of

80 amino acids diffuse out of the parasitized erythrocyte into the external milieu [13-15]. The relative

81 amounts of amino acids released correlate well with their abundance in hemoglobin [13, 14]. Thus, it

82 appears that many of the amino acids generated during the catabolism of hemoglobin exit the parasite.

83 We hypothesized that measurement of the rate of release of amino acids from blood-stage $P$.

84 falciparum could serve as the basis for a non-invasive and sensitive readout of flux through the

85 hemoglobin endocytic-catabolic pathway. Such an assay could bolster efforts to study endocytic process

86 in P. falciparum and to identify and characterize the effects of inhibitors of hemoglobin endocytosis and 
87 catabolism in vivo. Existing methods are either insufficiently sensitive to probe the kinetics of

88 hemoglobin endocytosis and/or catabolism over short (30 minutes to 3 hours) timeframes, or are difficult

89 to scale up to medium- to high-throughput capacity. For example, polyacrylamide gel-based approaches

90 to monitor the accumulation of undegraded hemoglobin in P. falciparum have been employed to

91 characterize the in vivo effects of cysteine protease inhibitors, quinoline- and artemisinin-family anti-

92 malarials and actin-perturbing agents on hemoglobin endocytosis and catabolism [16-20]. These methods

93 can deliver quantitative data over short time periods [16]; however they are not readily scalable to

94 medium- or high-throughput capacity. Quantitation of inhibition of endocytosis has typically relied on the

95 uptake from resealed erythrocytes of endocytic tracers such as biotin- or fluorophore-labeled dextran or

96 horse radish peroxidase [3, 17, 18]. While this approach has yielded valuable insights, it suffers from the

97 significant disadvantage that the resealing process necessarily alters the composition of the host

98 erythrocyte cytosol, including the concentration of hemoglobin, which likely affects rates of hemoglobin

99 endocytosis and catabolism.

100 In this report, we describe a convenient, sensitive and non-invasive method for quantitation of

101 the flux through the hemoglobin endocytic/catabolic pathway at high temporal resolution. We

102 demonstrate the utility of this approach by characterizing the effects of wortmannin and

103 dihydroartemisinin (DHA), two inhibitors of $P$. falciparum phosphatidylinositol-3-kinase, on the kinetics

104 of hemoglobin endocytosis and catabolism in vivo.

105

106 2. MATERIALS AND METHODS

107 2.1. P. falciparum culture and formulation of amino acid-restricted media

$108 \quad$ P. falciparum clone $3 \mathrm{D} 7$ was routinely cultured in human $\mathrm{O}^{+}$erythrocytes (Interstate Blood Bank;

$1092 \%$ hematocrit) in RPMI 1640 medium (Life Technologies) supplemented with $27 \mathrm{mM}$ sodium

110 bicarbonate, $11 \mathrm{mM}$ glucose, $0.37 \mathrm{mM}$ hypoxanthine, $10 \mu \mathrm{g} / \mathrm{mL}$ gentamicin, and $5 \mathrm{~g} / \mathrm{L}$ Albumax I

111 (Invitrogen). For all experiments, parasite cultures were maintained in a $5 \% \mathrm{CO}_{2}$ incubator at $37^{\circ} \mathrm{C}$. We

112 note that a reduced-oxygen gas was not used, as we have found that the 3D7 line replicates with a $\sim 4$ 
113 hour cycle at ambient oxygen levels in a 5\% $\mathrm{CO}_{2}$ environment. Parasites were synchronized by $5 \%$

114 sorbitol treatment [21].

115 A variation of RPMI containing isoleucine as the only proteinogenic amino acid ("Ile+Nva") was

116 formulated as previously described ([12]; Table 1). The medium designated "RPMI-VL+Nva" contained

117 all proteinogenic amino acids except Ala, Leu and Val at the concentrations indicated in Table 1. In both

118 media, $100 \mu \mathrm{M} D$-norvaline (Nva) was added to serve as an internal standard.

\subsection{Amino acid efflux assays}

Synchronized, mid-trophozoite stage parasites (one to two nuclei, $\sim 28$ hours post-invasion) were

122 used for all efflux assays. Parasites at 5-10\% parasitemia (infected RBC, or iRBC) or uninfected

123 erythrocytes (uRBC) were washed three times with amino-acid restricted medium and aliquotted into 96-

124 well plates $(200 \mu \mathrm{L}$ per well, $2 \%$ hematocrit). At the prescribed times, aliquots were removed to a

125 separate plate and centrifuged at $860 \times \mathrm{g}$ for 3 minutes. The conditioned medium was removed and stored

126 at $4{ }^{\circ} \mathrm{C}$ until the end of the experiment, at which time all media samples were transferred to an Ultracel-10

127 MultiScreen filter plate (10 kDa cutoff, Millipore) and centrifuged at $1940 \mathrm{x} g$ for 30 minutes. The flow-

128 through, depleted of proteins, was progressed to amino acid analysis or frozen at $-20{ }^{\circ} \mathrm{C}$.

130 Chromatography (UPLC) system equipped with an Acquity UPLC BEH C18 column (2.1 x 100 mm, 1.7

$131 \mu \mathrm{m}$ particle size). Mobile phases were AccQ-Tag Ultra Eluent A and B (Waters) and the column

132 temperature was $55{ }^{\circ} \mathrm{C}$. Programs for the separation of all amino acids in Ile+Nva medium or for the rapid 133 quantitation of Leu and D-norvaline in RPMI-VL+Nva are provided in Tables $\mathrm{S} 1$ and S2, respectively.

134 Pre-column derivatization was conducted by mixing $5 \mu \mathrm{L}$ of filtered conditioned medium with $15 \mu \mathrm{L}$ of

$135500 \mathrm{mM}$ sodium borate $\mathrm{pH} 9.5$ and $5 \mu \mathrm{L}$ of AccQ-Tag reagent (6-aminoquinolyl- $N$-hydroxysuccinimidyl

136 carbamate, [22]; Waters). Control experiments indicated that the quantity of amino acids in RPMI-

137 VL+Nva fell within the linear range of the assay. After one minute, samples were heated to $55^{\circ} \mathrm{C}$ for 10

138 minutes to convert a labile di-derivative of Tyr to the mono-derivative [22]. Injection volume was $1 \mu \mathrm{L}$ 
139 and derivatized amino acids were detected at $260 \mathrm{~nm}$. Peak areas were determined by manual integration

140 using Empower2 software (Waters). Concentrations of amino acids in media were calculated by reference

141 to the peak area of the $100 \mu \mathrm{M} D$-norvaline internal standard.

142 To determine amino acid efflux rates, aliquots of medium were collected after 2, 4, 6 and 8 hours

143 incubation. Rates were determined by linear regression fit to a concentration vs. time plot (e.g., Fig. 1B).

144 The first two hours were considered to be an equilibration period and were not included in the fit. To

145 normalize amino acid efflux rates to parasite numbers, aliquots of $P$. falciparum culture and uninfected

146 erythrocyte controls were fixed by mixing with an equal volume of $0.2 \%$ glutaraldehyde in phosphate

147 buffered saline. After permeabilization with $0.25 \%$ Triton X-100, samples were incubated with $400 \mathrm{nM}$

148 YOYO-1 (Life Technologies) in phosphate buffered saline. Parasite density per unit volume was

149 measured using an Accuri C6 flow cytometer (BD Biosciences) with manual gating to define the

150 population of infected erythrocytes. Background was determined from samples of uninfected

151 erythrocytes.

152 The effect of E64-d on amino acid efflux was determined in a four-hour endpoint assay.

153 Immediately after washing into amino acid-restricted medium, $10 \mu \mathrm{M}$ E64-d or 0.1\% DMSO was added

154 to the cultures. Four hours later, medium was collected and prepared for analysis of amino acid

155 concentrations as described above.

\section{2.3. Parasite growth rate assays}

158 Asynchronous parasites were washed into the following media: regular RPMI (section 2.1);

159 RPMI-VL+Nva (Table 1); RPMI-VL (identical to RPMI-VL+Nva except $D$-norvaline was absent); and

160 RPMI-VL supplemented with $200 \mu \mathrm{M}$ Leu and $171 \mu \mathrm{M}$ Val, designated RPMI-VL+VL. Every two days

161 for 8 days, an aliquot was taken for determination of parasitemia by flow cytometry as described in

162 section 2.2 and a separate aliquot was subcultured. The natural log of the product of parasitemia and

163 cumulative dilution factor was plotted against time in hours and fit by linear regression to the equation

$164 \ln (\mathrm{p})=\ln (\mathrm{a})+\ln (2) \bullet t / \tau$, where $\mathrm{p}=$ parasitemia $\mathrm{x}$ cumulative dilution factor, $\mathrm{a}=$ starting parasitemia and $\tau$ 
$165=$ doubling time. For each medium, mean doubling times and standard deviations were calculated from

166 three separate growth rate determinations in the same experiment.

\subsection{Validation of D-norvaline as an internal standard}

iRBC (16\% parasitemia, trophozoite stage; $2 \%$ hematocrit) and uRBC (2\% hematocrit) cultures

170 were washed into RPMI-VL+Nva and divided into twelve $200 \mu \mathrm{L}$ aliquots in a 96-well culture plate. In

171 addition, twelve $200 \mu \mathrm{L}$ aliquots of medium alone were added to the plate. Samples were incubated for

172 four hours, at which time media were collected, filtered and derivatized as described in section 2.2.

173 Amino acids were resolved using the UPLC program in Table S2 and areas of the Ile, Leu and Nva peaks

174 were determined by manual integration. For each analyte, mean peak area and standard deviation were

175 calculated from the values obtained for the twelve samples. Statistical analysis was conducted using a

176 two-tailed Student's t-test.

\subsection{Wortmannin and dihydroartemisinin inhibition of Leu efflux}

iRBC cultures were washed into RPMI-VL+Nva, supplemented with $10 \mu \mathrm{M}$ wortmannin (EMD

\section{RESULTS AND DISCUSSION}

\subsection{Chromatographic separation of amino acids}

Methods to resolve and quantify pre-column derivatized amino acids by high-pressure liquid chromatography have been available for many years. A factor limiting their application has been the long

188 run times that were required to resolve all of the proteinogenic amino acids. The recent development of

189 ultra-high pressure liquid chromatography (UPLC) and sub-2 $\mu \mathrm{m}$ particle columns has brought the time

190 of amino acid analysis down to less than ten minutes while improving sensitivity and peak resolution 
191 [23]. We investigated the potential of this technology to rapidly quantify amino acid levels in conditioned

192 medium from $P$. falciparum cultures. Using standard solutions of amino acids, we found that of the

193 twenty proteinogenic amino acids (including both reduced and oxidized cysteine), all were resolved

194 except Arg and Gln (Table 2). We also determined the retention times of ornithine and citrulline, two

195 metabolites of Arg that have been shown to accumulate in culture medium [24], of 4-hydroxyproline, and

196 of methionine sulfoxide and methionine sulfone, two oxidation products of Met (Table 2). Ornithine, 4-

197 hydroxyproline and Met sulfone were resolved from the proteinogenic amino acids; however, citrulline

198 co-eluted with Glu. Attempts to resolve these two amino acids by modifying the chromatography

199 conditions were not successful. In addition, Met sulfoxide co-eluted with Asp. In summary, under the

200 UPLC conditions used here, we are able to quantify the levels of all proteinogenic amino acids except

201 Arg, Gln, Asp and Glu.

202

2033.2 Characterization of amino acid efflux by cultured $\mathrm{P}$. falciparum trophozoites

204 Our studies of amino acid efflux focused on the trophozoite stage of the asexual cycle, as this is

205 when the rate of hemoglobin uptake and catabolism is at its peak [1]. To quantify efflux rates,

206 synchronized P. falciparum 3D7 trophozoites and uninfected erythrocyte controls were washed into an

207 amino acid-restricted, RPMI-based medium that contained $L$-Ile as the only proteinogenic amino acid

208 [12]; $100 \mu \mathrm{M} D$-norvaline was included in the medium as an internal standard to permit accurate

209 quantitation of effluxed amino acids (I+Nva medium, Table 1; see section 3.3 for validation of $D$ -

210 norvaline as an internal standard). Chromatograms of amino acids released after 8 hours by iRBC and

211 uRBC are shown in Fig. 1A. Both URBC and iRBC released amino acids into the medium; however, for

212 most of these, the amounts of amino acids effluxed from iRBC greatly exceeded those of uRBC. In the

213 analyses that follow, iRBC efflux rates are corrected for the contribution of uRBC to the observed

214 concentration of amino acid in the medium.

215 The efflux of amino acids from synchronized trophozoite cultures was highly linear over eight

216 hours, which permitted the determination of efflux rates by linear regression (Fig. 1B). To permit 
217 comparisons between experiments, efflux rates were normalized to parasite numbers (determined by flow 218 cytometry) and were reported as $\mathrm{nmol} / \mathrm{h} / 10^{7}$ parasites (Table 2 ). This approach afforded highly

219 reproducible efflux rates, as indicated by the relatively small standard deviations from three independent 220 biological replicates (Table 2). A comparison of efflux rates for all amino acids exhibiting rates over 0.2 $221 \mathrm{nmol} / \mathrm{h} / 10^{7}$ parasites (the estimated lower limit for reliable quantitation) is shown in Fig. 1C. The non222 polar amino acids Ala, Val and Leu exhibited the highest efflux rates. Substantial rates of efflux were also 223 observed for Gly, Phe, Pro, Ser, Thr and His. Efflux rates were low but measurable for Tyr, Trp, Asn, Lys 224 and ornithine (the latter was presumably derived from effluxed Arg [24]). Negligible/no efflux was 225 detected for the sulfur-containing amino acids Cys, cystine, and Met. Efflux rates were not determined for 226 Asp, Glu, Arg and Gln due to incomplete resolution from other species (Table 2); however, inspection of 227 chromatograms suggests that parasites efflux very little of these amino acids (Fig. 1A). The efflux rate for 228 Ile could not be determined because it is a component of the culture medium. Because hemoglobin catabolism has been reported to make a major contribution to amino acid 230 efflux $[13,14]$, we asked whether the rates of the more highly effluxed amino acids ( 9 amino acids with 231 efflux rates of $\geq 1 \mathrm{nmol} / \mathrm{h} / 10^{7}$ parasites) correlate with their abundance in human $\alpha$ - and $\beta$-globin (Fig. 232 1D). The coefficient of determination $\left(R^{2}\right)$ value for a linear regression fit was used as a measure of the 233 correlation between the two variables. There was a strong correlation between efflux rates and amino acid 234 abundance in $\alpha$ - and $\beta$-globin, with an $\mathrm{R}^{2}$ value of 0.90 . To determine whether this correlation simply 235 reflects general trends in the use of amino acids in proteins, we examined the correlation of efflux rates 236 with amino acid abundance in human myoglobin. Myoglobin is distantly related to hemoglobin and has a 237 similar oxygen-binding function. The correlation was much weaker with myoglobin, with an $\mathrm{R}^{2}$ value of 238 0.29. We also examined the correlation with amino acid abundance in the UniProt database release 239 2014_5, which is derived from 228,536 protein sequences. Here too, the correlation between efflux rates 240 and abundance was poor (Fig. 1D). These results are consistent with erythrocyte hemoglobin serving as a 241 dominant source of the effluxed amino acids included in the analysis. We note, however, that the 
242 correlation breaks down for amino acids such as Asp and Lys; these residues are effluxed at much lower

243 rates (if at all) than would be predicted by their abundance in human hemoglobin. Thus, there appears to

244 be an element of selectivity in the efflux of amino acids from the parasitized erythrocyte.

245 To further examine whether hemoglobin is the source of effluxed amino acids, the effect of 10

$246 \mu \mathrm{M}$ E-64d on amino acid concentrations in the medium was assessed. E-64d is a cell-permeant cysteine

247 protease inhibitor that blocks the proteolytic activity of food vacuole falcipains and causes the

248 accumulation of undegraded hemoglobin in the vacuole [25]. In a four-hour endpoint assay, E-64d

249 reduced the concentrations of amino acids in the medium to about $20 \%$ of control values (Fig. 1E), which

250 is consistent with hemoglobin catabolism serving as the dominant source of effluxed amino acids. The

251 residual level of amino acids observed after four hours could accrue from incomplete falcipain inhibition,

252 from hemoglobin catabolism by other vacuolar endopeptidases such as the plasmepsins, or from

253 contributions by other proteolytic pathways, such as the proteasome.

254 Finally, we asked whether parasites efflux a substantial fraction of the amino acids generated

255 from hemoglobin catabolism. Val and Leu were used for this analysis because they are effluxed at high

256 rates and the parasite lacks biosynthetic pathways for these amino acids. Using an adult human mean

257 corpuscular hemoglobin value of $30 \mathrm{pg} / \mathrm{cell}$ [26] and the efflux rates for Val and Leu in Table 2, and

258 assuming that these rates are sustained for a third of the 44 hour asexual cycle during which $70 \%$ of

259 erythrocyte hemoglobin is degraded [1], we calculate that $41 \%$ of Val and $40 \%$ of Leu generated from

260 hemoglobin catabolism is effluxed. This exercise suggests that a large fraction of the amino acids

261 generated through hemoglobin catabolism is lost from the parasite and is consistent with a previous report

262 that asexual P. falciparum uses only one-fifth of the amino acids generated from hemoglobin catabolism

263 for protein synthesis [2].

2653.3 Reformulation of the amino acid composition of RPMI medium for sensitive and accurate quantitation 266 of Leu and Val efflux 
Taken together, our studies indicate that amino acids that are both highly abundant in hemoglobin 268 and that are effluxed from parasitized erythrocytes at high rates (e.g., Ala, Leu, Val) can be useful probes 269 of in vivo flux through the hemoglobin endocytic-catabolic pathway. Because Ala does not elute near the $270 D$-norvaline internal standard (Fig. 1A), we focused on Leu and Val as the analytes of choice. The use of

271 two analytes rather than one provides redundancy, which could be advantageous when conducting 272 inhibitor screens; should a screening compound interfere with one of the analytes, the other will be 273 available for quantitation. In this section, we describe the development of a robust and rapid assay for 274 quantifying Leu and Val efflux.

275 While Ile+Nva medium was useful for profiling global amino acid efflux in the above 276 experiments, we have observed that many laboratory P. falciparum lines grow more slowly in this 277 medium, and is was reported that some lines such as HB3 fail to grow at all [12]. To develop an amino 278 acid efflux assay that can be used with the widest range of parasite lines, we reformulated the standard 279 RPMI recipe such that it contained all proteinogenic amino acids except Ala, Val and Leu (regular RPMI 280 lacks Ala but contains Val and Leu; Table 1). In addition to removing these amino acids, the 281 concentrations of many other amino acids were reduced such that the highest concentration of any amino 282 acid was $200 \mu \mathrm{M}$ (Table 1). The rationale for these changes was to reduce the total amine content of the 283 medium to avoid exceeding the linear range of the assay. The concentration of Ile was reduced from 381 $284 \mu \mathrm{M}$ to $100-150 \mu \mathrm{M}$ to enhance baseline separation between Ile and the neighboring Leu peak. Finally, the 285 non-proteinogenic amino acid 4-hydroxyproline, a component of regular RPMI, was omitted. The 286 reformulated medium was named "RPMI-VL" to reflect the removal of Val and Leu.

287 An internal standard was considered to be necessary to account for evaporation of cultures during 288 incubation and for variability introduced during pipetting and sample injection steps. $D$-norvaline 289 appeared to be a promising candidate based on its structural similarity to the analytes, its resolution from 290 all proteinogenic amino acids (Table 2) and its proximity in the chromatogram to the analytes Val and 291 Leu (Fig. 1A). The $D$-isomer was chosen to avoid any potential toxic effects arising from 
292 misincorporation into proteins [27]. $100 \mu \mathrm{M} D$-norvaline was added to RPMI-VL; this medium was 293 termed "RPMI-VL+Nva".

294 To determine the whether these changes to the standard RPMI formula had any effect on parasite

295 viability or cell cycle duration, growth rate assays were carried out with P. falciparum clone 3D7.

296 Doubling times in the following media were determined: i) standard RPMI; ii) RPMI-VL; iii) RPMI-VL

297 supplemented with $200 \mu \mathrm{M}$ Leu and $171 \mu \mathrm{M}$ Val (designated RPMI-VL+VL); and iv) RPMI-VL+Nva.

298 Growth in the reformulated media was as robust as it was in standard RPMI (Fig. 2A). Thus, there

299 appeared to be no deleterious effect on proliferation rate arising from the removal of Leu and Val or the

$300 \quad$ addition of $D$-norvaline.

301 To be an effective internal standard, $D$-norvaline levels should not change appreciably in the

302 presence of a high parasite load. To test whether this was the case, peak areas for $D$-norvaline, Leu and Ile

303 in RPMI-VL+Nva were determined after four hours incubation of: i) medium alone; ii) medium

304 containing P. falciparum-infected erythrocytes at 16\% parasitemia, 2\% hematocrit; and iii) medium

305 containing uninfected erythrocytes at 2\% hematocrit (Fig. 2B). As expected, high levels of Leu were only

306 observed in the iRBC sample. Consumption of Ile, which is not present in human hemoglobin, was also

307 observed only for the iRBC sample and was consistent with the use of this amino acid for parasite protein

308 synthesis. Levels of $D$-norvaline were very similar in the three samples, but did appear to be elevated by

309 about 5\% in the iRBC samples. Assuming a normal distribution and equal variance, this difference was

310 statistically significant, with a $p$-value $<0.001$ for the difference in the means of the iRBC and media

311 alone samples. The reason for this slight elevation is not known; it is possible that a small amount of an

312 unknown parasite metabolite co-elutes with $D$-norvaline. In most cases, this small deviation will be

313 inconsequential. We examined the possibility of using $\alpha$-aminoadipic acid as an alternate internal

314 standard; however, this additive had a slight suppressive effect on parasite growth (data not shown) and

315 was not further pursued.

316

317

3.4. Effect of wortmannin and dihydroartemisinin on Leu efflux 
319 inhibition of hemoglobin endocytosis and catabolism, we selected wortmannin as a challenging test case.

320 Wortmannin is a potent and selective inhibitor of phosphatidylinositol-3-kinases (PI3Ks), enzymes that

321 catalyze a phosphotransfer reaction from ATP to phosphatidylinositol to generate phosphatidylinositol-3-

322 phosphate (PI3P) [28]. Wortmannin has been shown to inhibit P. falciparum PI3K in vitro and to

323 substantially reduce parasite synthesis of PI3P [29, 30]. However, wortmannin is highly unstable in RPMI

324 medium, forming adducts with a number of amino acids that have reduced inhibitory activity [31].

325 Perhaps because of this lability, we have found that wortmannin at $10 \mu \mathrm{M}$ concentration does not impair

326 parasite proliferation, despite genetic evidence that PI3K is an essential enzyme [29]. The inability of

327 wortmannin to kill parasites at single doses as high as $25 \mu \mathrm{M}$ has been previously reported [29]; in

328 contrast, wortmannin was found to "stall" parasite growth over six days of treatment [30].

In mammalian and yeast cells, PI3P is predominantly located on early endosomes and plays

330 critical roles in endocytosis, endosome fusion and motility, and protein traffic to the vacuole or lysosome

331 [32]. In P. falciparum, PI3P has been localized to the membranes of the food vacuole, the apicoplast and

332 the lumen of the endoplasmic reticulum $[29,33]$. The presence of PI3P on the food vacuole membrane

333 implies a role in the transport of endocytosed hemoglobin. Consistent with this idea, wortmannin has been

334 shown to impair hemoglobin delivery to the vacuole. Vaid et al have reported that a five-hour incubation

335 with wortmannin caused an accumulation of undegraded hemoglobin in the parasite, which appeared to

336 be contained in vesicular structures [30]. In a separate study, wortmannin promoted food vacuole

337 fragmentation and disruption of the vacuolar membrane [34]. On the basis of these findings, a reduction

338 in the rate of hemoglobin catabolism was expected upon wortmannin treatment.

339 To determine whether Leu efflux could serve as an indicator of wortmannin-induced alterations

340 in rates of hemoglobin uptake and catabolism in vivo, iRBC were washed into RPMI-VL+Nva and

341 supplemented with either $0.1 \%$ DMSO (control) or $10 \mu \mathrm{M}$ wortmannin. Culture media were sampled

342 every 30 minutes for six hours and concentrations of Leu were determined. Suppression of Leu efflux by

343 wortmannin was evident after 60 minutes and continued for the duration of the experiment (Fig. 3A). 
344 Interestingly, there appeared to be two phases to the kinetics of wortmannin inhibition of Leu efflux (Fig.

345 3B). In the first phase (" 1 " in Fig. 3B), lasting from 1 to 2.5 hours, the rate of Leu efflux in the presence 346 of wortmannin was reduced to a steady-state value that was $15 \%$ of that in the control (Fig. 3C). Over

347 time, however, the rate of Leu efflux in the presence of wortmannin increased, reaching a value

348 corresponding to half that of the control in the time period of 5 - 6 hours (Fig. 3C). These data support a

349 role for PI3K-catalyzed de novo PI3P synthesis in hemoglobin endocytosis and delivery to the food

350 vacuole; however, they also suggest that wortmannin inhibition of PI3K is transient, likely due to the

351 instability of wortmannin in culture medium [31]. The "recovery" Leu efflux kinetics that we observe

352 may be due to the biosynthesis of PI3K once wortmannin is exhausted from the medium, and they

353 provide an explanation for the lack of wortmannin lethality when administered in single doses [29].

354 A recent study has revealed that $P$. falciparum $\mathrm{PI} 3 \mathrm{~K}$ is potently inhibited by the artemisinin class

355 of anti-malarials [35]. To further define the consequences of PI3K inhibition on flux through the

356 hemoglobin endocytic/catabolic pathway, we compared the effects of wortmannin and dihydroartemisinin

357 (DHA) on Leu efflux (Fig. 3D). DHA was selected for this analysis because its effects on both parasite

358 PI3K activity (complete inhibition at $4 \mathrm{nM})$ and $3 \mathrm{D} 7$ parasite replication $\left(\mathrm{EC}_{50}\right.$ value of $5 \mathrm{nM}$ ) have been

359 characterized $[35,36]$. Leu efflux profiles for parasites incubated with wortmannin $(10 \mu \mathrm{M})$ and DHA (50

360 and $500 \mathrm{nM}$; 10-fold and 100-fold above the $\mathrm{EC}_{50}$ value, respectively) both reveal strong suppression of

361 Leu efflux between one and two hours. However, after two hours, the rate of Leu efflux in the presence or

362 wortmannin begins to increase, while in the presence of DHA it maintains a steady-state value that is well

363 described by a linear fit of the data from 1-5 hours. Comparison to the DMSO control indicates that the

364 steady state rates of Leu efflux are reduced to $10 \%$ and $2 \%$ of control upon treatment with $50 \mathrm{nM}$ and 500

365 nM DHA, respectively. These results are consistent with a common mode of action (i.e., inhibition of

366 PI3K) by wortmannin and DHA, with the sustained effect of the latter likely arising from its greater

367 stability.

368 Together, these experiments provide important "proof-of-principle" evidence that Leu efflux can

369 provide a detailed kinetic picture of the in vivo perturbation of hemoglobin endocytosis and/or catabolism 
by small molecules. This assay should prove valuable in the characterization of inhibitors that are

371 expected to target these processes.

\section{CONCLUSIONS}

A sensitive and precise method with high temporal resolution has been developed to interrogate the kinetics of amino acid efflux from cultured P. falciparum. Use of amino acid-restricted culture media, an internal standard, and ultra-high pressure liquid chromatography were key factors in enabling rapid and accurate quantitation of effluxed amino acids. The utility of this method for characterizing the in vivo inhibition of hemoglobin uptake (wortmannin, DHA) and catabolism (E-64d) was demonstrated. We anticipate that this approach will provide valuable insights into the mechanism of action of compounds that are predicted to perturb hemoglobin endocytosis or catabolism. It should also find application in screens for novel inhibitors of these processes.

\section{FIGURE LEGENDS}

Figure 1: Characterization of amino acid efflux by $\boldsymbol{P}$. falciparum trophozoites. (A) UPLC chromatograms of amino acids in Ile+Nva medium after an eight hour incubation with iRBC (black) or uRBC (red). C-C, cystine; Cit, citrulline; oxMet; Met sulfoxide; O, ornithine; oxGlut, oxidized glutathione; Nva, $D$-norvaline. The asterisk indicates a peak originating from the derivatization reagent. Peak assignments were inferred from amino acid standards. (B) Rates of amino acid efflux to the culture medium are highly linear over eight hours. Lines indicate linear regression fits to the data points. (C) Comparison of mean efflux rates for 13 proteinogenic amino acids and ornithine $(\mathrm{O})$, which is likely not effluxed but rather derived from extracellular Arg. Error bars are standard deviations from three independent biological replicates. (D) Relationship between amino acid abundance and efflux rates of selected amino acids for human $\alpha$ - and $\beta$-globin (top panel), human myoglobin (middle panel) and the UniProt database (lower panel). Lines indicate linear regression fits. (E) Inhibition of amino acid efflux upon treatment with $10 \mu \mathrm{M}$ E-64d for four hours. Inhibition is reported as percentage of a $0.1 \%$ DMSO control. Error bars are standard deviations from three independent biological replicates.

Figure 2: Validation of reformulated RPMI and the use of $\boldsymbol{D}$-norvaline as an internal standard. (A) Doubling times of 3D7 parasites in regular RPMI and in RPMI-VL, RPMI-VL supplemented with Val and Leu (RPMI-VL+VL), and RPMI-VL with $D$-norvaline (RPMI-VL+NVA) as assessed in a 6-day growth experiment. Error bars are the standard deviation for three separate doubling time determinations in one experiment. (B) Comparison of peak areas for Ile, Leu and $D$-norvaline in the absence of added cells ("medium") or in the presence of uRBC ( $2 \%$ hematocrit) or iRBC ( $2 \%$ hematocrit, $16 \%$ parasitemia) after 4 hours. Bars are the mean values obtained from analysis of twelve samples (see section 2.4). Error bars indicate the standard deviation. 
Figure 3: Inhibition of Leu efflux by PI3K inhibitors. (A) Time course of Leu efflux from iRBC treated with $10 \mu \mathrm{M}$ wortmannin (circles) or $0.1 \%$ DMSO (triangles) and from uRBC (squares). Data points are the average of triplicate analyses (iRBC) or represent single analyses (uRBC). Similar results were obtained in two other independent experiments (not shown). (B) Wortmannin inhibition of Leu efflux is transient. The same data as in (A) are shown but with an expanded ordinate. Two kinetic phases are shown: an early inhibition phase ("1"; 1 to $2.5 \mathrm{~h}$ ) and a later recovery phase ("2"; 5 to $6 \mathrm{~h}$ ). Lines were generated by linear regression fits. (C) Rates of Leu efflux calculated from the data in panels A and B. $\mathrm{iRBC} / \mathrm{DMSO}$ and $\mathrm{URBC}$ rates were determined by linear regression fit of data from $0-6 \mathrm{~h}$.

$\mathrm{iRBC} /$ wortmannin rates were determined as shown in panel B. (D) Comparison of effects of wortmannin and dihydroartemisinin (DHA) on Leu efflux. Leu effluxed by uRBC has been subtracted from data points. Lines were generated by linear regression fits of data from 1-5 h.

\section{ACKNOWLEDGMENTS}

420 This work was supported by grants from the National Institutes of Health (AI077638), the Bill \& Melinda

421 Gates Foundation (OPP1067465) and USDA National Institute of Food and Agriculture, project VA-

422 139761. The sponsors had no role in study design, collection and interpretation of data, writing of the

423 manuscript or decision to publish. We are grateful to Waters Corp. for excellent technical support.

\section{REFERENCES}

426 [1] Hanssen E, Knoechel C, Dearnley M, Dixon MW, Le Gros M, Larabell C, et al. Soft X-ray microscopy analysis of cell volume and hemoglobin content in erythrocytes infected with asexual and

428 sexual stages of Plasmodium falciparum. J Struct Biol 2012;177:224-32.

429 [2] Krugliak M, Zhang J, Ginsburg H. Intraerythrocytic Plasmodium falciparum utilizes only a fraction of the amino acids derived from the digestion of host cell cytosol for the biosynthesis of its proteins. Mol Biochem Parasitol 2002;119:249-56.

[3] Abu Bakar N, Klonis N, Hanssen E, Chan C, Tilley L. Digestive-vacuole genesis and endocytic

434 [4] Aikawa M, Hepler PK, Huff CG, Sprinz H. The feeding mechanism of avian malarial parasites. J Cell 435 Biol 1966;28:355-73.

436 [5] Elliott DA, McIntosh MT, Hosgood HD, Chen S, Zhang G, Baevova P, et al. Four distinct pathways of hemoglobin uptake in the malaria parasite Plasmodium falciparum. Proc Natl Acad Sci USA

438 2008;105:2463-8.

439 [6] Lazarus MD, Schneider TG, Taraschi TF. A new model for hemoglobin ingestion and transport by the 440 human malaria parasite Plasmodium falciparum. J Cell Sci 2008;121:1937-49.

441 [7] Francis SE, Sullivan DJ, Jr., Goldberg DE. Hemoglobin metabolism in the malaria parasite 442 Plasmodium falciparum. Annu Rev Microbiol 1997;51:97-123. 
443 [8] Milani KJ, Schneider TG, Taraschi TF. Defining the morphology and mechanism of the hemoglobin transport pathway in Plasmodium falciparum-infected erythrocytes. Eukaryot Cell 2015;14:415-26.

445 [9] Dalal S, Klemba M. Roles for two aminopeptidases in vacuolar hemoglobin catabolism in 446 Plasmodium falciparum. J Biol Chem 2007;282:35978-87.

447 [10] Rosenthal PJ. Hydrolysis of erythrocyte proteins by proteases of malaria parasites. Curr Opin 448 Hematol 2002;9:140-5.

449 [11] Goldberg DE. Hemoglobin degradation. Curr Top Microbiol Immunol 2005;295:275-91.

450 [12] Liu J, Istvan ES, Gluzman IY, Gross J, Goldberg DE. Plasmodium falciparum ensures its amino acid 451 supply with multiple acquisition pathways and redundant proteolytic enzyme systems. Proc Natl Acad Sci 452 USA 2006;103:8840-5.

453 [13] Cenedella RJ, Rosen H, Angel CR, Saxe LH. Free amino-acid production in vitro by Plasmodium 454 berghei. Am J Trop Med Hyg 1968;17:800-3.

455 [14] Zarchin S, Krugliak M, Ginsburg H. Digestion of the host erythrocyte by malaria parasites is the 456 primary target for quinoline-containing antimalarials. Biochem Pharmacol 1986;35:2435-42.

457 [15] Groman NB. Dynamic aspects of the nitrogen metabolism of Plasmodium gallinaceum in vivo and in 458 vitro. J Infect Dis 1951;88:126-50.

459 [16] Famin O, Ginsburg H. Differential effects of 4-aminoquinoline-containing antimalarial drugs on 460 hemoglobin digestion in Plasmodium falciparum-infected erythrocytes. Biochem Pharmacol $461 \quad 2002 ; 63: 393-8$.

462 [17] Hoppe HC, van Schalkwyk DA, Wiehart UI, Meredith SA, Egan J, Weber BW. Antimalarial 463 quinolines and artemisinin inhibit endocytosis in Plasmodium falciparum. Antimicrob Agents Chemother 464 2004;48:2370-8.

465 [18] Roberts L, Egan TJ, Joiner KA, Hoppe HC. Differential effects of quinoline antimalarials on 466 endocytosis in Plasmodium falciparum. Antimicrob Agents Chemother 2008;52:1840-2.

467 [19] Rosenthal PJ. Plasmodium falciparum: effects of proteinase inhibitors on globin hydrolysis by 468 cultured malaria parasites. Exp Parasitol 1995;80:272-81.

469 [20] Smythe WA, Joiner KA, Hoppe HC. Actin is required for endocytic trafficking in the malaria 470 parasite Plasmodium falciparum. Cell Microbiol 2008;10:452-64.

471 [21] Lambros C, Vanderberg JP. Synchronization of Plasmodium falciparum erythrocytic stages in 472 culture. J Parasitol 1979;65:418-20.

473 [22] Cohen SA, Michaud DP. Synthesis of a fluorescent derivatizing reagent, 6-aminoquinolyl-N474 hydroxysuccinimidyl carbamate, and its application for the analysis of hydrolysate amino acids via high475 performance liquid chromatography. Anal Biochem 1993;211:279-87.

476 [23] Salazar C, Armenta JM, Cortes DF, Shulaev V. Combination of an AccQ·Tag-Ultra Performance 477 Liquid Chromatographic Method with Tandem Mass Spectrometry for the Analysis of Amino Acids. In: 
Alterman MA, Hunziker P, editors. Amino Acid Analysis: Methods and Protocols: Springer Science; 2012. p. 13-28.

480 [24] Olszewski KL, Morrisey JM, Wilinski D, Burns JM, Vaidya AB, Rabinowitz JD, et al. Host-parasite interactions revealed by Plasmodium falciparum metabolomics. Cell Host Microbe 2009;5:191-9.

[25] Rosenthal PJ. Cysteine proteases of malaria parasites. Int J Parasitol 2004;34:1489-99.

[26] Vajpayee N, Graham SS, Bem S. Basic examination of blood and bone marrow. In: McPherson RA, Pincus MR, editors. Henry's clinical diagnosis and management by laboratory methods. 22 ed: Saunders; 2011. p. 509-35.

[27] Apostol I, Levine J, Lippincott J, Leach J, Hess E, Glascock CB, et al. Incorporation of norvaline at leucine positions in recombinant human hemoglobin expressed in Escherichia coli. J Biol Chem 1997;272:28980-8.

[28] Ui M, Okada T, Hazeki K, Hazeki O. Wortmannin as a unique probe for an intracellular signalling protein, phosphoinositide 3-kinase. Trends Biochem Sci 1995;20:303-7.

[29] Tawk L, Chicanne G, Dubremetz JF, Richard V, Payrastre B, Vial HJ, et al. Phosphatidylinositol 3phosphate, an essential lipid in Plasmodium, localizes to the food vacuole membrane and the apicoplast.

493 Eukaryot Cell 2010;9:1519-30.

494 [30] Vaid A, Ranjan R, Smythe WA, Hoppe HC, Sharma P. PfPI3K, a phosphatidylinositol-3 kinase from 495 Plasmodium falciparum, is exported to the host erythrocyte and is involved in hemoglobin trafficking.

496 Blood 2010;115:2500-7.

497 [31] Yuan H, Barnes KR, Weissleder R, Cantley L, Josephson L. Covalent reactions of wortmannin under 498 physiological conditions. Chemistry \& biology 2007;14:321-8.

499 [32] Raiborg C, Schink KO, Stenmark H. Class III phosphatidylinositol 3-kinase and its catalytic product 500 PtdIns3P in regulation of endocytic membrane traffic. Febs J 2013;280:2730-42.

501 [33] Bhattacharjee S, Stahelin RV, Speicher KD, Speicher DW, Haldar K. Endoplasmic reticulum PI(3)P 502 lipid binding targets malaria proteins to the host cell. Cell 2012;148:201-12.

503 [34] Howe R, Kelly M, Jimah J, Hodge D, Odom AR. Isoprenoid biosynthesis inhibition disrupts Rab5

504 localization and food vacuolar integrity in Plasmodium falciparum. Eukaryot Cell 2013;12:215-23. mechanism of artemisinin resistance in Plasmodium falciparum malaria. Nature 2015;520:683-7. 


\title{
Amino acid efflux by asexual blood-stage Plasmodium falciparum and its utility in interrogating the kinetics of hemoglobin endocytosis and catabolism in vivo
}

\author{
Seema Dalal and Michael Klemba* \\ Department of Biochemistry, Virginia Tech, Blacksburg, VA, USA 24061
}

A facile, non-invasive method to quantify flux through the hemoglobin endocyticcatabolic pathway via amino acid efflux is described.

Time course of amino acid efflux by cultured Plasmodium falciparum

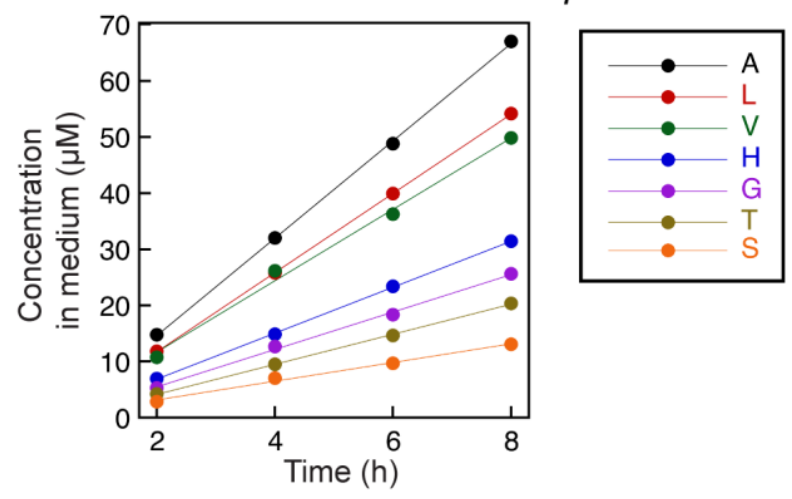


$A_{20} \longrightarrow B$

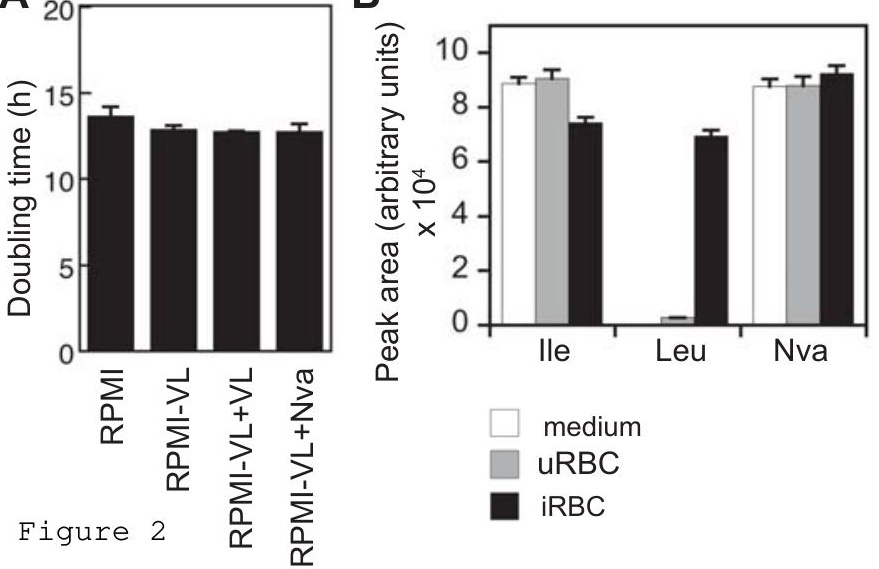


